\title{
Dabigatran must be used carefully: literature review and recommendations for management of adverse events
}

This article was published in the following Dove Press journal:

Drug Design, Development and Therapy

\author{
Shan Lin* \\ Yan Wang* \\ Lei Zhang \\ Wei Guan
}

Department of Respiratory Medicine, Qinghai University Affiliated Hospital, Xining 81000I, People's Republic of China

*These authors contributed equally to this work
Correspondence: Wei Guan

Department of Respiratory Medicine, Qinghai University Affiliated Hospital, Xining 81000I, People's Republic of China Tel +860 971 6230883

Email weiguanII0@I63.com

\begin{abstract}
Atrial fibrillation increases the risk of stroke and death. The vitamin-K antagonist warfarin is recommended for patients with atrial fibrillation, but vitamin- $\mathrm{K}$ antagonists are cumbersome to use. Therefore, an effective, safe and convenient new anticoagulant is needed. Dabigatran acts by inhibiting free and fibrin-bound thrombin directly. It is an oral anticoagulant that was approved by the US Food and Drug Administration. The oral anticoagulant dabigatran has been used increasingly due to its good tolerance, predictable pharmacokinetics, effective anticoagulant effects, and absence of requirement of coagulation monitoring. However, an increasing prevalence of adverse events has been reported, some of them quite serious. Therefore, we searched and reviewed the literature on dabigatran with regard to adverse events, and proposed solutions to prevent and reduce the chance of adverse events occurring.
\end{abstract}

Keywords: adverse events, dabigatran, allergic reactions, bleeding, esophageal injury

\section{Introduction}

Atrial fibrillation increases the risk of stroke and death. ${ }^{1}$ The vitamin-K antagonist warfarin is recommended for patients with atrial fibrillation and who are at risk of stroke, which reduces the risk of stroke and death, but increases the risk of bleeding. Vitamin-K antagonists are cumbersome to use due to: interactions with multiple foods and drugs; a narrow therapeutic range; significant changes in anticoagulant response; a slow onset of action; requirement of frequent testing of laboratory parameters. Patients who receive warfarin have poor compliance and a high prevalence of treatment withdrawal due to the factors mentioned above, so many patients do not receive adequate anticoagulant therapy. ${ }^{2}$ Therefore, an effective, safe and convenient new anticoagulant is needed.

Dabigatran acts by inhibiting free and fibrin-bound thrombin directly. It is an oral anticoagulant that was approved by the US Food and Drug Administration in 2010 to prevent embolic events in patients with non-valvular atrial fibrillation. ${ }^{3}$ Dabigatran has been used increasingly in clinical practice due to its good tolerance, predictable pharmacokinetics, effective anticoagulant effects, and absence of need of coagulation monitoring. Dabigatran is used as an alternative to warfarin, and it appears to be as effective as warfarin in preventing embolic events in patients with non-hemorrhagic stroke and atrial fibrillation rather than in patients with valve problems. $^{4}$ 


\section{Adverse events of dabigatran}

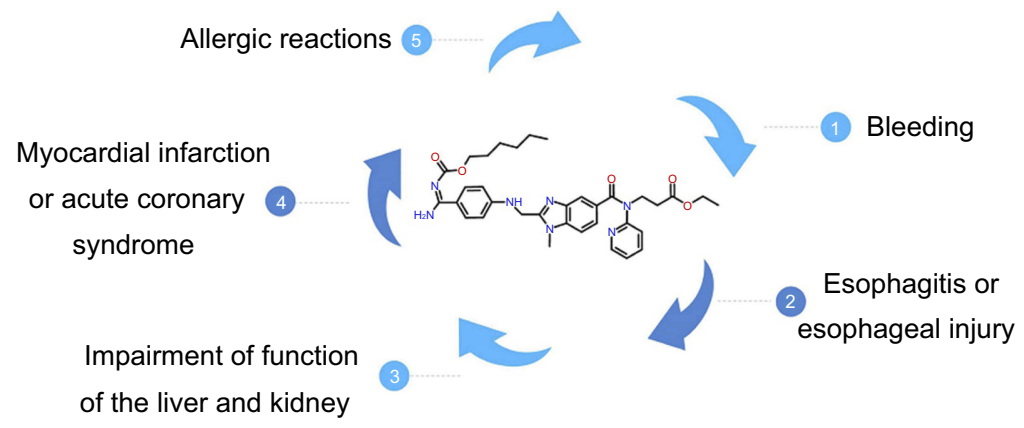

Figure I The adverse events of dabigatran.

Despite the many advantages of dabigatran, reports of adverse events have emerged in recent years, such as gastrointestinal discomfort and bleeding. Although patients taking dabigatran have fewer life-threatening hemorrhages, the prevalence of gastrointestinal bleeding was significantly higher than in those who used warfarin. ${ }^{3}$ An increasing prevalence of adverse events has been reported, ${ }^{5-27}$ some of them quite serious. Therefore, we searched and reviewed the literature on dabigatran with regard to adverse events (Figure 1).

We searched PubMed, Google scholar and the Chinese National Knowledge Infrastructure using the key words "dabigatran and adverse events", "dabigatran and side effects" and "dabigatran and induced" from December 2010 to November 2018. Case reports were selected on the adverse events of dabigatran that they described. Twenty-three case reports were reviewed and analyzed, and the clinical features are listed in Table 1.

\section{Adverse events of dabigatran Bleeding}

As with any anticoagulant drug, various types of hemorrhage are common adverse events. Of the cases we reviewed, seven patients developed severe hemorrhage, ${ }^{5-11}$ including three cases of fatal gastrointestinal hemorrhage. ${ }^{8-10}$ Major bleeding was reported to occur in a dose-dependent manner, and this was reported in fewer than $3 \%$ of patients during a trial comparing dabigatran with other anticoagulants. ${ }^{28,29}$ Similarly, Connolly et al reported that the prevalence of major bleeding was $2.71 \%$ per year in the group receiving dabigatran (110 mg, b.d.) and 3.11\% per year in the group receiving dabigatran $(150 \mathrm{mg}$, b.d.), and that the prevalence of life-threatening bleeding in those receiving 110 and $150 \mathrm{mg}$ of dabigatran twice-daily was $1.22 \%$ and $1.45 \%$, respectively. ${ }^{3}$
The three patients who died of gastrointestinal bleeding caused by dabigatran were old (mean age $=81$ years). One of them took only $150 \mathrm{mg}$ of dabigatran once-daily, ${ }^{8}$ but this may have been related to the hip arthroplasty that she underwent. These gastrointestinal events may have been due to the dabigatran formulation, which contains granules of tartaric acid to provide an acidic environment. A lower $\mathrm{pH}$ is associated with dyspepsia and plays a part in the increased risk of gastrointestinal bleeding. ${ }^{28}$ Spontaneous hemopericardium, ${ }^{5}$ spontaneous hemothorax ${ }^{6}$ and hemorrhagic cystitis $^{7}$ occurred separately in three patients. Major bleeding in these anatomic areas is uncommon and has rarely been reported, but it should be documented. Other risk factors of dabigatran-induced gastrointestinal bleeding include concurrent use of ulcerogenic drugs, older age, Helicobacter pylori infection, and previous history of gastrointestinal bleeding. Helicobacter pylori infection and use of NSAIDs are independent risk factors for duodenal ulcer and gastric ulcer and related bleeding. ${ }^{30,31}$ Therefore, we should prevent the combined hemorrhagic effect of dabigatran and Helicobacter pylori, and the patient should be screened for Helicobacter pylori before anticoagulant therapy. In general, these findings also show that the risk of venous thromboembolism and bleeding should be considered before deciding the anticoagulation given to the patient.

\section{Esophagitis or esophageal injury}

Another adverse event that requires attention is esophagitis or esophageal injury (including esophageal ulcers) induced by dabigatran intake. ${ }^{12-19}$ In 1983 , it was estimated that the esophageal injury caused by drugs was only 3.9 cases per 100,000 people per year. ${ }^{32}$ In 2016, Toya et al reported that dabigatran use caused $\sim 20 \%$ of patients to suffer damage to the esophageal mucosa. ${ }^{33}$ However, among 


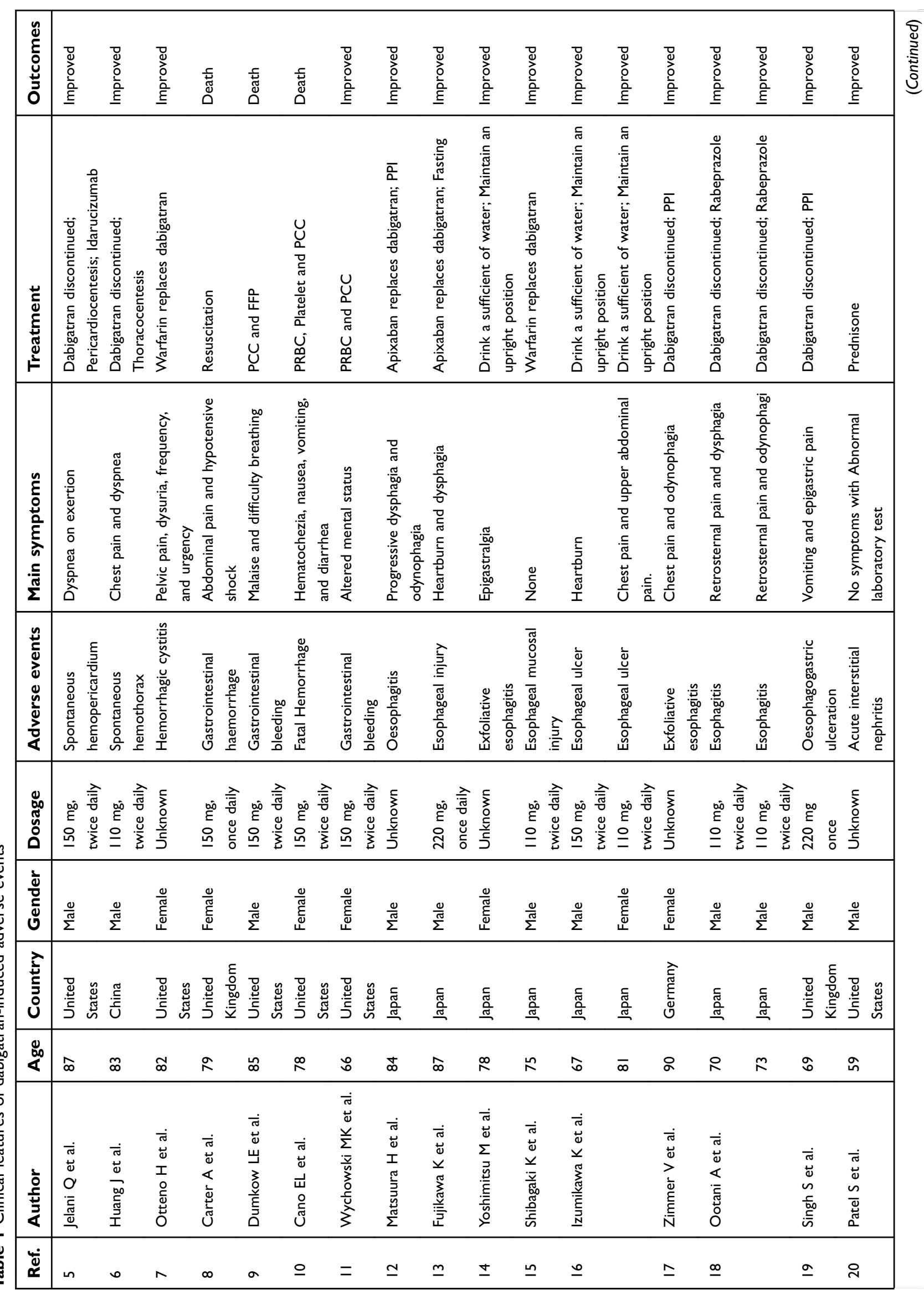




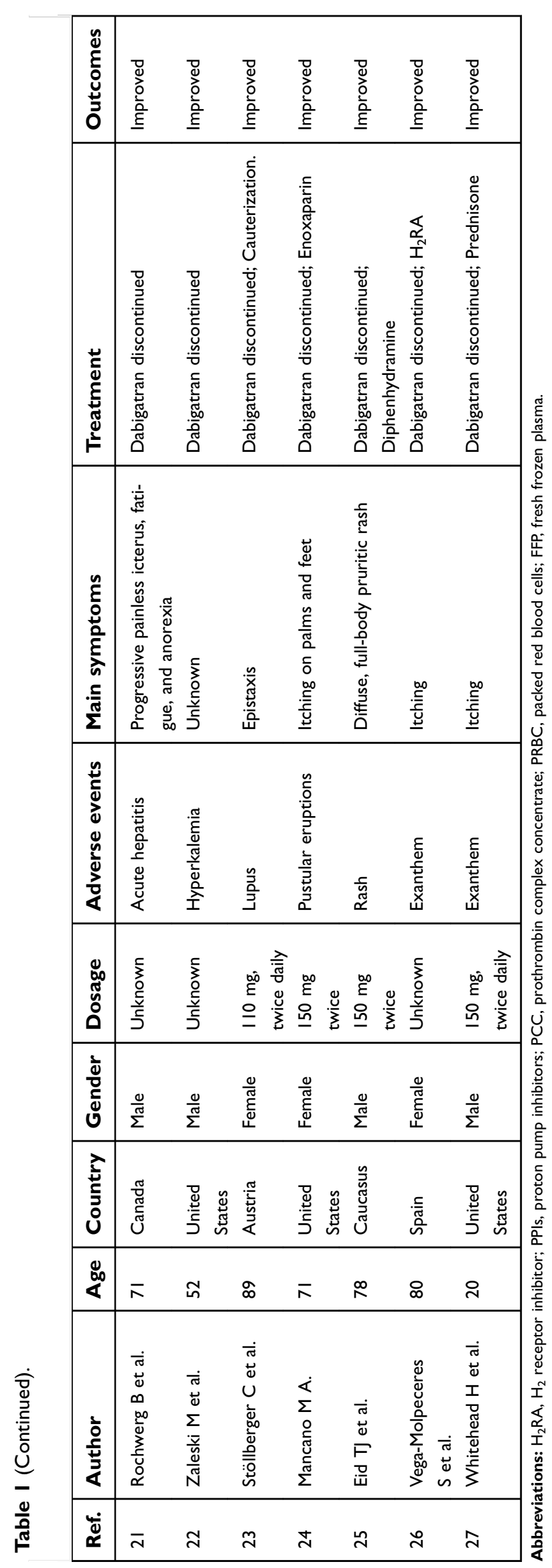


the cases we reviewed, 10 of 25 patients developed varying degrees of esophagitis or esophageal injury after dabigatran use. Therefore, dabigatran-induced esophagitis may be overlooked in asymptomatic patients who have not undergone endoscopy. Among the 10 patients (four males and six females) we reviewed, the mean age was 77 years and there was no significant difference between the doses of dabigatran used. It has been speculated that the tartaricacid core in dabigatran not only plays a part in gastrointestinal bleeding, but is released after digestion and adheres to the esophagus to damage the esophageal mucosa, and then the damaged esophageal mucosa exfoliates after peristalsis. ${ }^{34}$ In older patients, due to reduced activity (sitting or lying down for long periods) and reduced salivary secretion, if the volume of water consumed is low, the lack of a sufficient liquid bolus will increase the possibility of contact by dabigatran with the esophageal mucosa. The mean age of the patients we reviewed was 77 years, so we speculate that advanced age was an important risk factor for dabigatran-induced esophagitis. Clinicians should not ignore the possibility of esophagitis after giving patients dabigatran, especially if patients develop dysphagia, chest pain, upper abdominal pain, or heartburn.

\section{Impairment of function of the liver and kidney}

The Randomized Evaluation of Long-Term Anticoagulation Therapy (RE-LY) trial showed that impairment of liver function (an increase in the levels of aspartate aminotransferase or alanine aminotransferase by more than three times the upper limit of the normal range) caused by dabigatran did not occur more frequently than that with warfarin. ${ }^{3}$ In the literature that we reviewed, dabigatran-induced acute interstitial nephritis, ${ }^{20}$ acute hepatitis $^{21}$ and hyperkalemia ${ }^{22}$ were reported. In a phaseII dose-finding trial of dabigatran, Eriksson et al found only mild increases in transaminase levels in the dose ranges tested, but two patients had severe hepatitis (transaminase levels more than five times the upper limit of normal). ${ }^{35}$ The cause of liver and kidney dysfunction caused by dabigatran may be because first-pass elimination occurs in the liver and $80 \%$ of a given dose is excreted by the kidney. ${ }^{36}$ One of the patients in a study experienced hyperkalemia. Hyperkalemia is a potentially fatal side-effect that can occur in patients with altered renal function, and understanding that dabigatran can cause hyperkalemia in this patient population is crucial. Dabigatran can induce hyporeninemic hypoaldosteronism in patients with impaired renal function, which leads to hyperkalemia. Careful monitoring of renal function is recommended if physicians prescribe dabigatran for patients with renal insufficiency.

\section{Myocardial infarction or acute coronary syndrome}

A small (but significant) increased risk of myocardial infarction or acute coronary syndrome has been noted when combining safety-outcome data from multiple trials. $^{37}$ The RE-LY trial showed that the prevalence of myocardial infarction per year was $0.53 \%$ with warfarin, and that the prevalence per year in patients taking $110 \mathrm{mg}$ of dabigatran and $150 \mathrm{mg}$ of dabigatran was $0.72 \%$ and $0.74 \%$, respectively. ${ }^{3}$ These findings were probably because warfarin provides better protection against coronary ischemic events than dabigatran. ${ }^{38}$ In the literature that we reviewed, such adverse events were not reported in any patient. Nevertheless, clinicians should continue to consider the possibility of these severe, harmful cardiovascular effects after dabigatran administration.

\section{Allergic reactions}

Five patients developed allergic reactions after taking dabigatran. $^{23-27}$ In addition, one patient developed druginduced lupus (DIL). ${ }^{23}$ Stöllberger and colleagues suggested that a small lipophilic molecule with a molecular weight of 472 Da may play a part in immune side-effects after dabigatran administration. ${ }^{23}$ Furthermore, DIL is characterized by high levels of antinuclear antibodies and antihistone antibodies, with no evidence of complement consumption, and circulating immune complexes within the normal range. The RE-LY trial reported that fewer than $0.1 \%$ of patients receiving dabigatran reported drug allergies, allergic edema, allergic reactions, or anaphylactic shock. ${ }^{3}$ Even though allergic edema and allergic reactions are rare, monitoring and reporting allergic reactions in patients taking dabigatran is important.

\section{Age}

Among the patients with adverse events that we reviewed (except for a 20-year-old patient with a dabigatran-induced exanthema), the mean age was 76 (range, 52-90) years. ${ }^{5-27}$ Regardless of whether adverse events are associated with age, more clinical trials are needed to ascertain the relationship between age, dose, and adverse effects to better guide management. 


\section{Conclusions and recommendations}

The oral anticoagulant dabigatran is used widely. Nevertheless, clinicians cannot ignore the adverse events caused by dabigatran. We recommend the management detailed below for the adverse events caused by dabigatran.

(a) According to the patient's symptoms, signs and laboratory tests, once the diagnosis of gastrointestinal bleeding is clear, depending on the amount of bleeding, if a small amount of bleeding, in addition to observing clinical changes, the clinicians may consider adding $\mathrm{H}_{2}$ receptor inhibitor $\left(\mathrm{H}_{2} \mathrm{RA}\right)$, proton pump inhibitors (PPIs) and mucosal protective agent. In severe bleeding, measures include discontinuation of dabigatran immediately and administration of prothrombin complex concentrate, packed red blood cells, and fresh frozen plasma, the use of specific reversal agents such as idarucizumab for dabigatran, and emergency endoscopic management.

(b) If heartburn, chest pain and upper abdominal pain appear shortly after dabigatran administration, drinking a sufficient volume of water $(150 \mathrm{~mL}$ or more) and maintaining an upright position for $\geq 30$ mins are useful strategies for relieving symptoms. If necessary, PPIs and $\mathrm{H}_{2} \mathrm{RAs}$ can be added. If symptoms persist, dabigatran should be discontinued; apixaban, rivaroxaban and warfarin are alternatives.

(c) If patients have dysphagia, upper abdominal pain, chest pain, retrosternal pain, vomiting, and odynophagia, and their duration is long, clinicians should be alert to the possibility of dabigatran-induced esophagitis or esophageal injury. Endoscopy should be undertaken as soon as possible. Longitudinal exfoliation of the distal esophagus is a characteristic feature of dabigatran-induced esophagitis.

(d) If abdominal pain, anorexia, or fatigue is the main clinical manifestations of dabigatran use, hepatic lesions should be considered after excluding esophageal lesions. Monitoring indicators of liver function are particularly important. If hepatic lesions are present, dabigatran should be stopped and drugs that can protect liver function, such as bicyclol, glutathione, and magnesium isoglycyrrhizinate, should be administered.

(e) Impairment of renal function (including acute interstitial nephritis and hyperkalemia) should be noted, especially hyperkalemia. Discontinuation of dabigatran and laboratory tests is recommended. Hemofiltration and glucocorticoid therapy are feasible if indicated.

(f) As an anticoagulant, dabigatran, may increase the risk of myocardial infarction or acute coronary syndrome. Clinicians should consider the possibility of these severe, harmful cardiovascular effects following use of dabigatran.

(g) Allergic reactions such as rash are not very common and can be treated with antihistamines, and discontinue dabigatran if necessary. DIL due to dabigatran is extremely rare but, if it occurs, dabigatran should be discontinued as soon as possible and autoantibody profiles are helpful for the diagnosis.

\section{Acknowledgments}

Shan Lin's deepest gratitude goes first and foremost to Professor Wei Guan, Shan Lin's supervisor, for her constant encouragement and guidance. She guided Shan Lin along with all those who helped during the writing of this thesis. Without her consistent and illuminating instruction, this thesis could not have reached its present form. This review was funded by QingHai Department of Science and Technology, the Grant Number: 2017-wjzd-11.

\section{Disclosure}

All authors claim no conflicts of interest in this work.

\section{References}

1. Kannel WB, Wolf PA, Benjamin EJ, Levy D. Prevalence, incidence, prognosis, and predisposing conditions for atrial fibrillation: population-based estimates. Am J Cardiol. 1998;82(7):2N-9N.

2. Brass L, Krumholz L, Scinto J, et al. War farin use following ischemic stroke among medicare patients with atrial fibrillation. Arch Intern Med. 1998;158:2093-2100.

3. Connolly SJ, Ezekowitz MD, Yusuf S, et al. Dabigatran versus warfarin in patients with atrial fibrillation. $N$ Engl J Med. 2009;361 (12):1139-1151. doi:10.1056/NEJMoa0905561

4. Gómez-Outes A, Terleira-Fernández AI, Calvo-Rojas G, et al. Dabigatran, rivaroxaban, or apixaban versus warfarin in patients with nonvalvular atrial fibrillation: a systematic review and meta-analysis of subgroups. Semin Thromb Hemost. 2018.

5. Jelani Q, Gordon R, Schussheim A. Dabigatran-induced spontaneous hemopericardium and cardiac tamponade. Tex Heart Inst J. 2017;44 (5):370-372. doi:10.14503/THIJ-16-6073

6. Huang J, Lin W, Lv D, et al. Dabigatran-induced massive spontaneous hemothorax. Drug Saf Case Rep. 2017;4(1):12. doi:10.1007/s40800017-0056-x

7. Otteno H, Smith E, Huffaker RK. Dabigatran induced hemorrhagic cystitis in a patient with painful bladder syndrome. Case Rep Urol. 2014;2014. 
8. Carter A, Sarda P, George M, et al. Hip arthroplasty fatality related to dabigatran induced gastrointestinal haemorrhage. Ann R Coll Surg Engl. 2014;96(1):e01-e03. doi:10.1308/003588414X13824511649779

9. Dumkow LE, Voss JR, Peters M, Jennings DL. Reversal of dabigatran-induced bleeding with a prothrombin complex concentrate and fresh frozen plasma. Am J Health Syst Pharm. 2012;69 (19):1646-1650. doi:10.2146/ajhp120055

10. Wychowski MK, Kouides PA. Dabigatran-induced gastrointestinal bleeding in an elderly patient with moderate renal impairment. Ann Pharmacother. 2012;46(4):e10. doi:10.1345/aph.1Q747

11. Cano EL, Miyares MA. Clinical challenges in a patient with dabigatran-induced fatal hemorrhage. Am J Geriatr Pharmacother. 2012;10(2):160-163. doi:10.1016/j.amjopharm.2012.02.004

12. Matsuura H, Suto $K$, Yasuhara $H$, et al. Longitudinal sloughing mucosal casts: dabigatran-induced oesophagitis. Eur Heart J. 2018;39(36):3400. doi:10.1093/eurheartj/ehw180

13. Fujikawa K, Takasugi N, Goto T, et al. Very late-onset dabigatraninduced esophageal injury. Can J Cardiol. 2017;33(4):554.e15-554. e16. doi:10.1016/j.cjca.2016.12.017

14. Yoshimitsu M, Sawada T, Kobayashi T, Yamagishi M. Dabigatraninduced exfoliative esophagitis. Intern Med. 2016;55(13):1815. doi:10.2169/internalmedicine.55.6606

15. Shibagaki K, Taniguchi H, Goto D, Kobayashi K, Kinoshita Y. Dabigatran-induced asymptomatic esophageal mucosal injury. Gastrointest Endoac. 2016;83(2):472-474. doi:10.1016/j.gie.2015.08.028

16. Izumikawa $\mathrm{K}$, Inaba $\mathrm{T}$, Mizukawa $\mathrm{S}$, et al. Two cases of dabigatran-induced esophageal ulcer indicating the usefulness of drug administration guidance. Nihon Shokakibyo Gakkai zasshi. 2014;111(6):1096-1104.

17. Zimmer V, Casper M, Lammert F. Extensive dabigatran-induced exfoliative esophagitis harboring squamous cell carcinoma. Endoscopy. 2014;46(S 01):E273-E274. doi:10.1055/s-0034-1365782

18. Ootani A, Hayashi Y, Miyagi Y. Dabigatran-induced esophagitis. Clin Gastroenterol Hepatolthe. 2014;12(7):e55. doi:10.1016/j. cgh.2013.09.010

19. Singh S, Savage L, Klein M, et al. Case report: severe necrotic oesophageal and gastric ulceration associated with dabigatran. BMJ Case Rep. 2013;2013.

20. Patel S, Hossain MA, Ajam F, et al. Dabigatran-induced acute interstitial nephritis: an important complication of newer oral anticoagulation agents. J Clin Med Res. 2018;10(10):791. doi:10.14740/jocmr3569w

21. Rochwerg B, Xenodemetropoulos T, Crowther M, Spyropoulos A. Dabigatran-induced acute hepatitis. Clin Appl Thromb Hemost. 2012. doi:10.1177/1076029611435840

22. Zaleski M, Dabage N, Paixao R, Muniz J. Dabigatran-induced hyperkalemia in a renal transplant recipient: a clinical observation. J Clin Pharmacol. 2013;53(4):456-458. doi:10.1002/jcph.21

23. Stöllberger C, Krutisch G, Finsterer J, Wolf HM. Dabigatran-induced lupus temporarily preventing blood group determination. Blood Coagul Fibrinolysis. 2014;25(6):625-627. doi:10.1097/MBC.000000000 0000099
24. Mancano MA. ISMP adverse drug reactions: influenza vaccineinduced Stevens-Johnson Syndrome; vilazodone-induced nightmares; dabigatran-induced pustular eruptions; neurotoxic and cardiotoxic symptoms after cannabis concentrate exposure; rosuvastatin-induced skin eruption. Hosp Pharm. 2018;53(1):15-17. doi:10.1177/ 0018578717739727

25. Eid TJ, Shah SA. Dabigatran-induced rash. Am J Health Syst Pharm. 2011;68(16):1489-1490. doi:10.2146/ajhp110088

26. Vega-Molpeceres S, Just SM, Escoda GM. Dabigatran induced exanthema. Rev Clin Esp. 2010;210(11):590.

27. Whitehead H, Boyd JM, Blais DM, et al. Drug-induced exanthem following dabigatran. Ann Pharmacother. 2011;45(10):e53. doi:10.1345/aph.1P572

28. Blommel ML, Blommel AL. Dabigatran etexilate: a novel oral direct thrombin inhibitor. Am J Health Syst Pharm. 2011;68 (16):1506-1519. doi:10.2146/ajhp100348

29. Schulman S, Kearon C, Kakkar AK, et al. Dabigatran versus warfarin in the treatment of acute venous thromboembolism. $N$ Engl J Med. 2009;361(24):2342-2352. doi:10.1056/NEJMoa0906598

30. Lanas-Gimeno A, Lanas A. Risk of gastrointestinal bleeding during anticoagulant treatment. Expert Opin Drug Saf. 2017;16(6):673-685. doi:10.1080/14740338.2017.1325870

31. Pellicano R, Ribaldone DG, Fagoonee S, et al. A 2016 panorama of Helicobacter pylori infection: key messages for clinicians. Panminerva med. 2016;58(4):304-317.

32. Friedman AC, Oyewole MA. Pill-induced esophageal injury. Case reports and review of the medical literature. Dig Dis Sci. 1983;28:174-182.

33. Toya Y, Nakamura S, Tomita K, et al. Dabigatran-induced esophagitis: the prevalence and endoscopic characteristics. $J$ Gastroenterol Hepatol. 2016;31(3):610-614. doi:10.1111/jgh.13024

34. Bytzer P, Connolly SJ, Yang S, et al. Analysis of upper gastrointestinal adverse events among patients given dabigatran in the RE-LY trial. Clin Gastroenterol Hepatol. 2013;11(3):246-52. e5. doi:10.1016/j.cgh.2012.10.021

35. Eriksson B, Dahl O, Buller R, et al. A new oral direct thrombin inhibitor, dabigatran etexilate, compared with enoxaparin for prevention of thromboembolic events following total hip or knee replacement: the BISTRO II randomized trial. J Throm Haem. 2004;3 (1):103-111. doi:10.1111/j.1538-7836.2004.01100.x

36. Stangier J. Clinical pharmacokinetics and pharmacodynamics of the oral direct thrombin inhibitor dabigatran etexilate. Clin Pharmacokinet. 2008;47:285-295. doi:10.2165/00003088200847050-00001

37. Uchino K, Hernandez AV. Dabigatran association with higher risk of acute coronary events: meta-analysis of noninferiority randomized controlled trials. Arch Intern Med. 2012;172(5):397-402. doi:10.1001/archinternmed.2011.1666

38. Hurlen M, Abdelnoor M, Smith P, Erikssen J, Arnesen H. Warfarin, aspirin, or both after myocardial infarction. $N$ Engl J Med. 2002;347 (13):969-974. doi:10.1056/NEJMoa020496

\section{Publish your work in this journal}

Drug Design, Development and Therapy is an international, peerreviewed open-access journal that spans the spectrum of drug design and development through to clinical applications. Clinical outcomes, patient safety, and programs for the development and effective, safe, and sustained use of medicines are a feature of the journal, which has also been accepted for indexing on PubMed Central. The manuscript management system is completely online and includes a very quick and fair peer-review system, which is all easy to use. Visit http://www. dovepress.com/testimonials.php to read real quotes from published authors. 